\title{
The 3-step Greek protocol of neck skin rejuvenation inspired by a case of pseudoxanthoma elasticum: combination of laser skin resurfacing, collagen booster and PRP
}

\begin{abstract}
Aim: The physiological ageing process causes significant changes in the extracellular matrix $(\mathrm{ECM})$ of the neck skin, which are the first signs of ageing witness for women. We are inspired by a young woman, who suffers from a rare genetic disorder called pseudoxanthoma elasticum (PXE), which manifests irregular, thickened, fragmented and haphazardly orientated elastic fibers. We herein present a combination of three different therapies (laser skin resurfacing, type I horse collagen boosters and platelet rich plasma)as an approach to ameliorate neck skin sagging.
\end{abstract}

Methods: A 28-year-old Roma woman with a clinically diagnosed PXE, verified by ophthalmologic evidence and skin biopsy, underwent 3 sessions of this combined protocol every 3 weeks. One month after the last treatment session, a new biopsy was taken. During this period, the patient was encouraged for daily topical application of collagen mousse and vitamin C combined with sun block SPF50, along with limited exposure to sunlight.

Results: Clinical assessment supported by photographic documentation was performed at each session. Severe changes in hydration and tightening of the neck skin were obvious 3 weeks after the second session, although the verification of the results was substantiated with the pathology of the post treatment skin biopsy obtained 1 month following the completion of the protocol. The elastic fibers in the mid-dermis were dense, non-fragmented and parallel oriented. The aesthetic performance was evident until the final follow-up visit

Conclusions: Nowadays, the primary goal of aesthetic medical research is oriented to the dermis bio-reconstruction, where specific treatments try to improve the dermis quality from the inside to repair imperfections of skin ageing. The key of success is attributed to the best possible treatment combination capable of fibroblast stimulation.

Keywords: neck rejuvenation, pseudoxanthoma elasticum, PXE, collagen, laser resurfacing, $\mathrm{PRP}$
Volume 5 Issue 3 - 202I

Emmanouil Dimonitsas,' Agni Albanopoulou, ${ }^{2}$ Foteini Neamonitou, ${ }^{3}$ Ortansia Doryforou, ${ }^{4}$ Vasileios Liarakos, ${ }^{5}$ Irene Thymara, ${ }^{7}$ Raed Farhat, ${ }^{6}$ Penelope Korkolopoulou ${ }^{7}$

'Department of Plastic and Reconstructive Surgery, Greek Anticancer Institute, Greece

${ }^{2}$ Dermatologist-Chair of Skin Medical Secrets, Greece ${ }^{3}$ Department of General Surgery, The Royal of Wolverhampton NHS Trust, UK

${ }^{4}$ Chair of the Department of Ultrasound, 25 I General Airforce Hospital, Greece

${ }^{5}$ Consultant in the Department of Ophthalmology, Naval Hospital, Greece

'Plastic Surgeon-Chair of the Department of Wound Care, Plastic and Reconstructive Surgery, Al Zahra Hospital, United Arab Emirates

7Department of Pathology, National and Kapodistrian University of Athens, Greece

Correspondence: Emmanouil Dimonitsas, Deparment of Plastic and Reconstructive Surgery, Greek Anticancer Institute, Saint Savvas Hospital of Athens, Greece, Emailmdimonitsas@gmail.com

Received: June 01, 2021 | Published: June 14, 2021

\section{Background - pseudoxanthoma elasticum}

Pseudoxanthoma elasticum (PXE) is a rare inherited genetic disorder (autosomal dominant or recessive) characterized by fragmentation and calcification of elastic fibers with many systemic manifestations. Mutations in the ABCC6 gene, which is located to 16p13.1, have been identified in PXE. ${ }^{1}$ Recently, a second genetic locus, the ENPP1 gene has been described in patients with PXE-like clinical presentation. ${ }^{2}$

While the cutaneous manifestations are most apparent, other systems including the gastrointestinal, cardiovascular and ocular are typically involved. ${ }^{3}$ Onset is most often in the second decade, yet presentation in childhood has been documented with a progressive accumulation of mineral precipitates within the elastic fibers. ${ }^{4}$

The skin lesions consist of yellowish papules, relatively small (1-3mm), which tend to coalesce into larger plaques. These lesions may be accompanied by skin laxity, which results in redundant folds resembling "plucked-chicken skin" especially on flexural areas (antecubital, popliteal, inguinal, cervical, axillary folds and periumbilical). The involvement can be progressive and reach the entire skin and nearby mucosa (oral, vaginal and rectal). The affected skin gradually becomes redundant, lax, rigid and later wrinkled, particularly in the neck, axillary folds and thighs. ${ }^{5}$

Ophtalmoscopic investigation may also reveal the so-called angioid streaks, which can occur in $85 \%$ of patients but are not pathognomonic. These result from gaps in the elastic tissue of Bruch's membrane,which are situated between the choroid and pigment epithelium of the retina. Other findings from the eyes include hemorrhage, resulting in scarring or retinal detachment, mottled pigmentation (peau d'orange), subretinal neovascularization, peripapillary atrophy and optic disc drusen. ${ }^{6}$

Vascular involvement consists of degenerative changes of the internal and external elastic laminae, accompanied by intimal fibrosis and thickening. These changes result in weakness of the wall of media caliber arteries, with a tendency towards rupture and aneurysm formation together with calcification. Angina, hypertension, mitral incompetence and stenosis, aortic incompetence, aneurysm and intracerebral hemorrhage are described as well. ${ }^{7}$ Gastrointensal lesions result in hematemesis or melena and can be fatal with the stomach being the most affected site. ${ }^{8}$ 
According to the molecular, clinical and histopathological characteristics, specific criteria were established for the diagnosis of PXE (Table 1). Except for the pathognomonic ocular findings and typical skin lesions, confirmation is usually based on pathological examination of skin biopsy. Calcification of elastic fibers is identified with the von Kossa and the Alizarin red stains. ${ }^{9}$ The simultaneous presence of skin and eye involvement is required for the diagnosis of PXE; at the molecular level, two pathogenic ABCC6 mutations must exist. ${ }^{10}$

Table I Criteria for the diagnosis of PXE

\section{Definitive PXE*}

Two pathogenic mutations in the $A B C C 6$

OR

Ocular findings - angioid streaks $>$ I DD or peau d'orange in an individual $<20$ years of age

TOGETHER WITH

Skin findings

Characteristic pseudoxanthomatous papules and plaques on the neck or flexural creases,

Diagnostic histopathological changes in lesional skin: Calcified elastic fibers in the mid and lower dermis, confirmed by positive calcium stain

*Note that if definitive findings are present only in the skin or eyes, the presence of two pathogenic ABCC6 mutations revealed by subsequent genetic testing would confirm the diagnosis of PXE even in the absence of a complete phenotype.

\section{Possible PXE}

Without having met the above criteria, a patient could be considered to have "possible PXE", the degree of probability depending on the presence of other factors, including: family history and particularly affected siblings; microcalcifications in arterial blood vessels and other organs; histopathological changes in apparently unaffected skin; presence of a single PXE-associated mutation in either $A B C C 6$ or ENPPI, especially if only one mutation (the same) has been found in an affected sibling

\section{Treatment options}

Despite the continuous progress made in our understanding of the genetic basis of PXE, the pathomechanistic details leading from mutations in the ABCC6 gene to the ectopic mineralization and fragmentation of elastic fibres in the skin remain unclear. Thus, there is no treatment of choice for the systemic manifestations of PXE and a number of studies are at a preclinical level. ${ }^{3}$

Several studies conducted in mouse Abcc $6^{-/}$models are focused on the role of mineral composition of the diet as a potential modifier of the severity of PXE. These suggest that a high intake of dairy products, rich in calcium and phosphate, influences the extent of ectopic mineralization. Expressly, an increase of magnesium content or phosphate binders, limits the progression of cutaneous findings and vascular mineralization, whereas the increased calcium deposition deteriorates the disease. ${ }^{11}$

Moreover, bisphosphonates, pyrophosphate analogs (e.g. etidronate), used in generalized arterial calcification of infancy (GAGI) for counteracting the mineralization, may be helpful in PXE as well. PXE patients' fibroblasts experience an altered inorganic pyrophosphate (PPi) metabolism with reduced levels of PPi in their plasma and supplement of bisphosphonates improve the diminishing of PXE's progress. ${ }^{12}$ An alternative systemic treatment may involve the supplementation with fetuin-A, which permit the prevention of aberrant mineralization. ${ }^{13}$

Novel potential gene therapies for PXE are under development and are mainly liver-directed gene therapies. However, further research is needed to overcome several shortcomings of the gene therapy approach. $^{10}$

Intraocular injection of VEGF antagonist, laser-coagulation and photodynamic therapy can be used to counteract sub-retinal neovascularization and subsequent progressive blindness in patients suffering from PXE. ${ }^{14}$

\section{Treatment of skin manifestations of PXE}

Several topical retinoid therapies have been ulitilized with unsatisfactory results so far..$^{15}$ Skin lesions were usually treated with conservative cosmetic devices (cryotherapy, diatermocoagulation and $\mathrm{CO}_{2}$ laser-coagulation) to improve skin texture and prevent their progression. In isolated cases, fractional $\mathrm{CO}_{2}$ laser ablation was used cautiously, using low energy, and then adjusted step-by-step. The reaction of the PXE-affected skin was similar to that of the normal skin and results lasted 2 years with improvement of skin texture, surface irregularities and distensibility. ${ }^{16}$ Moreover, non-ablative lasers (e.g. Fraxel) has been used solitarily with reported evidence of collagen \& elastin enhancement and remodeling in the papillary dermis. ${ }^{17}$ Finally, injectable bovine collagen has already been tested for deep wrinkles of the lower jaw with immediately visible but temporary results. ${ }^{18}$

Rare reports for cosmetic improvement of PXE's skin manifestations suggest surgical management when implications exist e.g. presence of redundant, lax and indurated skin of the face and neck, with a typical "hound dog" appearance. Midface- and neck-lift include cutaneous rhytidectomy with SMAS (superficial musculoaponeurotic system) and is performed through a standard preauricular facelift incision with postauricular extension and transverse extension into the hairline, with impressive results and limited complications. ${ }^{19}$

\section{Ageing process of skin}

The physiological ageing process begins from our birth with a severe degradation of collagen and elastin production by the age of 25. The loss of the normal level of epidermis hydration, with the main component of the hyaluronic acid, is followed by major structural 
alterations of the extracellular matrix (ECM), consist of collagen and elastin fibers. ${ }^{20}$

Collagen fibers are a major component of the ECM, providing strength and skin elasticity. They account of $75 \%$ of the dry weight of skin with type I makes up $80-90 \%$ of the total collagen, while type III $8-12 \%$, and type $\mathrm{V}<5 \%$. In contrast to young skin, whereas collagen is abundant, tightly packed and well-organized, in aged skin collagen fibers are fragmented and coarsely distributed. ${ }^{21}$

Although elastin fibers correspond to only $2 \%$ of the total dermal protein,they play a significant role in skin compliance (the ability to be readily deformable) and resilience (the ability to recoil), which alltogether often referred as skin elasticity. In young skin, the elastic fibers adopt a characteristic highly ordered structure, with perpendicularly oriented fibrillin-rich microfibersat the papillary dermis. On the contrary, in aged skin, elastin microfibers are diminished and disorganised. ${ }^{22}$

Hyaluronic acid (HA) belongs to glycosaminoglycans (GAGs), which are large linear polysaccharides. It is mainly produced by fibroblasts, and is abundant in the papillary dermis. It crosslinks with other ECM proteins, like collagen, resulting in increased tissue stiffness, and plays space filling and shock-absorbing role. HA binding proteins (HABPs) play vital role in intracellular signaling pathways, regulating proliferation, migration, and differentiation. In aged skin, the HABPs are reduced compared to young skin, while the level of HA itself is not significantly different between young and old skin. ${ }^{23}$

Fibroblasts are the primary production cells, responsible for the collagen and elastin biosynthesis, which is a continuous process, and is balanced with the old fibers degradation by metalloproteinases (MMPs). The major source of MMPs is epidermal keratinocytes and dermal fibroblasts, although MMPs can also be produced by endothelial cells and immunocytes. ${ }^{24}$

Transforming growth factor- $\beta$ is an important regulator of ECM biosynthesis and homeostasis and controls the signaling pathway in dermal fibroblasts by enhancing ECM production. ${ }^{25}$

In young skin, fibroblasts adhere to the surrounding ECM and exert mechanical forces to maintain a normal elongated shape. In aged skin, fibroblasts attachment is impaired due to progressive ECM degradation, resulting in fibroblasts' size reduction, decreased elongation and collapsed morphology. The latter alterations along with the down regulation of TGF- $\beta$ signaling and the increase of MMPs levels regulate ECM degradation. ${ }^{26}$

Oxidative stress initiated by reactive oxygen species (ROS) increases MMPs' expression and inhibits TGF- $\beta$ signaling, which leads to decreased ECM biosynthesis. This concludes to an impaired adherence of fibroblasts to ECM, and consequently leads to the reduction of their size. The aged fibroblasts produce more ROS, accelerating in this way an endless positive cycle of dermal ageing. ${ }^{27}$

\section{Objective}

The aim of this study was to suggest a 3-step neck skin rejuvenation treatment protocol. We combined three different treatments: nonablative laser (Fraxel laser), intradermal collagen booster (NITHYA injectable product) and platelet-rich plasma (PRP) in order to produce new collagen and elastin in a young woman with a rare inherited disorder, pseudoxanthoma elasticum. The management of the cutaneous manifestations of PXE is focused on limiting the oxidative stress of the disease. The latter is responsible for the progressive calcification and fragmentation of elastic fibres, despite the existence of the underlying defects to the ABCC6 gene. ${ }^{2}$ Thus, inspired by this case, this anti-aging protocol can be generalized in order to be included as a new arrow in the quiver for neck skin rejuvenation.

\section{Materials and methods}

Fractional resurfacing is performed using a 1550/1927-nm non-ablative erbium/thulium fiber laser (Fraxel Dual; Reliant Technologies, Mountain View, CA) along with non-cross linked HA. This laser targets water-containing tissue to effect photocoagulation of narrow, sharply defined columns of skin known as microscopic thermal zones (MTZs). The local epidermis is preserved whereas the dermis column undergoes necrosis with formation of microscopic epidermal necrotic debris (MENDs). MENDs release their products from dermis to epidermis, leaving a dead space that will be filled by surrounded reservoir of spared epidermal and dermal tissue, allowing for rapid repair of laser-induced thermal injury and fiber synthesis. ${ }^{28}$

Nithya injectable product (manufactured and distributed by Euroresearch S.r.l. Milan - Italy) is a type III medical device containing type I horse collagen. It is in form of fine powder and is used diluted in $4.5 \mathrm{ml}$ saline and $0.5 \mathrm{ml}$ xylocaine. ${ }^{29}$

PRP preparation procedure includes peripheral blood collection in blood tubes containing $0.5 \mathrm{ml} \mathrm{3.2 \%} \mathrm{sodium} \mathrm{citrate} \mathrm{solution.} \mathrm{All} \mathrm{tubes}$ were being processed in a refrigerated angledcentrifuge (certified Nahita ECO 2640/80). The blood was centrifuged at 3630/rpm for 5 minutes and separated the platelets from the rest of the blood components. PRP is used for stimulation of both superficial and deep dermis layers. PRP is a bio-stimulator of fibroblasts, rich source of various cytokins and growth factors, which promotes cell proliferation, extracellular matrix synthesis and hyaluronic acid production. ${ }^{30}$

Except for these 3 steps, home-care cosmetic products included Nithya mousse and Version C 35\% serum. Nithya mousse (manufactured and distributed by Euroresearch S.r.1 Milan - Italy) is a medical device, which contains heterologous equine collagen at the concentration of $0.28 \% .{ }^{29}$ Version C $35 \%$ (manufactured and distributed by Version Dermocosmetics Athens- Greece)is the highest concentration of stabilized ascorbic acid (Vitamin C) in a low $\mathrm{pH}$ (3.0). It acts as an anti-oxidant protector of collagen, by inhibiting ROS species and MMPs production while it promotes fibroblasts proliferation and gene stimulation of pro-collagen. ${ }^{31}$

\section{Case description}

A 28-year-old Roma woman presented to the Dermatology Clinic in February 2020 for a longstanding progressive appearance of painless yellow papules with skin laxity in the cervical and axillary regions (Figure 1). These lesions remained unchanged with no medication or treatment. She had no pain complaints, or inflammatory signs on the lesions or around them, and did not have any associated symptoms. She did not take any daily medication and had no relevant personal or family medical history of dermatosis.

On examination, there were painless, uneven, rough, yellow plaques without inflammatory signs in the posterior cervical region. They merged bilaterally and symmetrically into the right and left side cervical region. Skin laxity was also presented in both axillary areas and abdomen. No other abnormalities were found on the rest of physical examination.

In the initial visit of the patient to the Dermatology clinic, the following exams were conducted: hemogram, general biochemistry 
with lipid profile and phosphorus-calcium balance, thyroid hormones testing and urinary sediment all of which were unremarkable. Further investigation with more specific examination included:
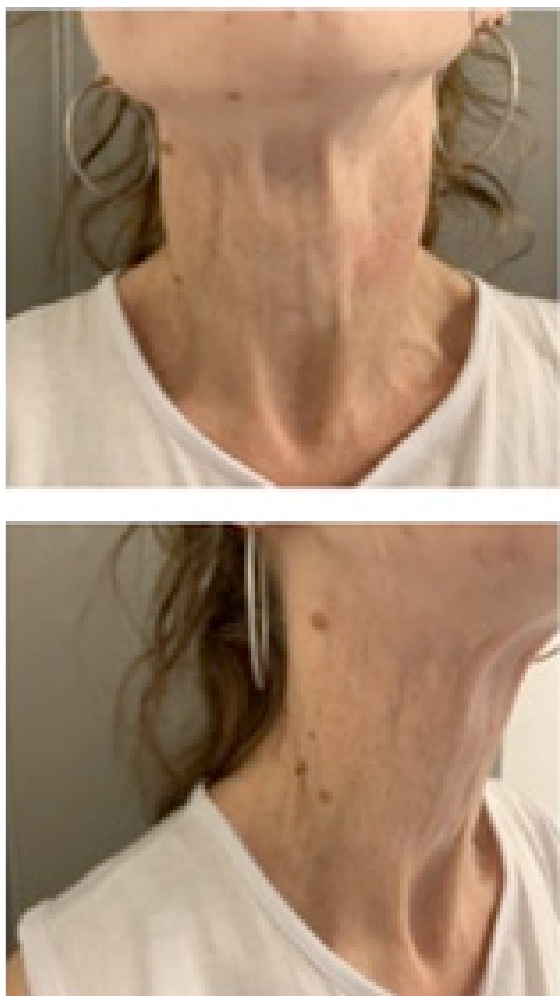

electrocardiogram and echocardiogram, breast ultrasound, carotid, aortoiliac venous and arterial ultrasonography.
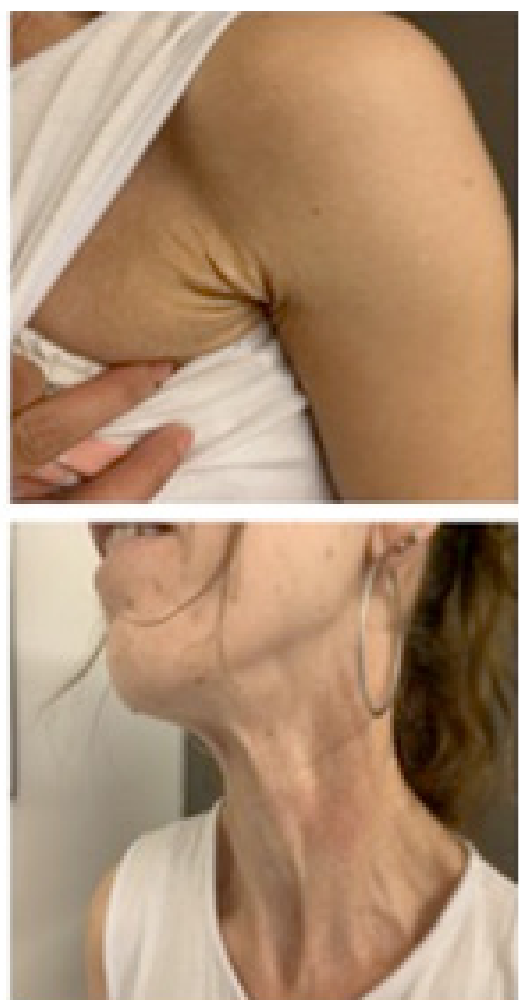

Figure I Clinical image of a young Roma woman with pseudoxanthoma elasticum.

Fundus images of the patient confirmed the presence of angioid streaks alongside with mottled pigmentation (peau d'orange) parafoveally, which are clearly visible in the right eye (Figure 2) and hardly visible in the left eye. Examination of the patient with highresolution 3-D Optical Coherence Tomography (OCT) showed no significant distortion in the area of the macula and the optic disks, apart from a relative asymmetry in the thickness of the Retinal Nerve Fiber Layer (RNFL) between the two eyes, with no further clinical impact. There was no sign of choroidal neovascularization (Figure 3).

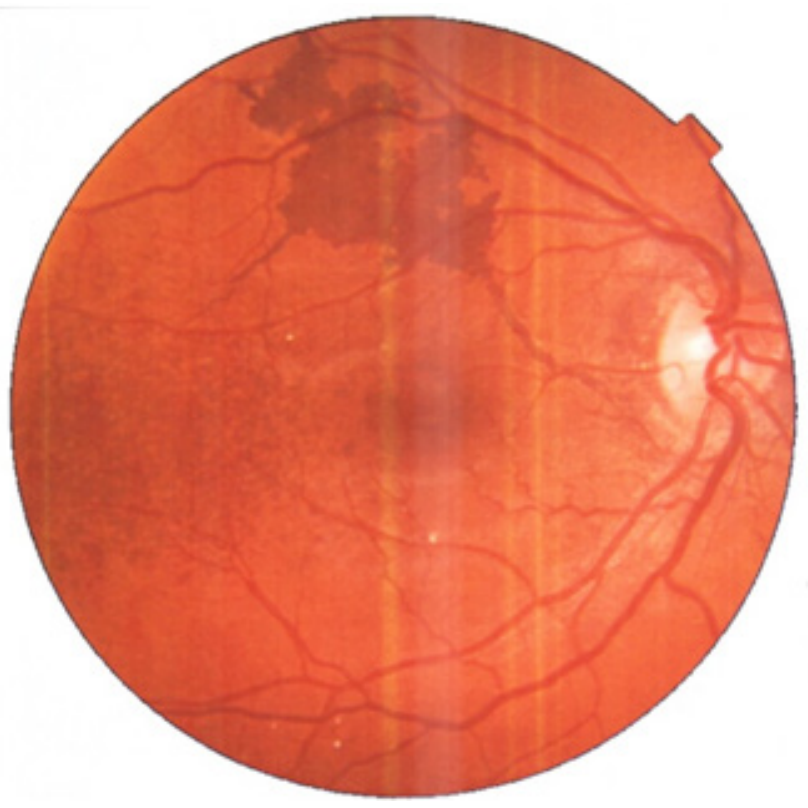

Figure 2 Fundus image of the right eye.

Citation: Dimonitsas E,Albanopoulou A, Neamonitou F, et al.The 3-step Greek protocol of neck skin rejuvenation inspired by a case of pseudoxanthoma elasticum: combination of laser skin resurfacing, collagen booster and PRP.J Dermat Cosmetol. 2021;5(3):66-73. DOI: 10.15406/jdc.202I.05.00 I86 


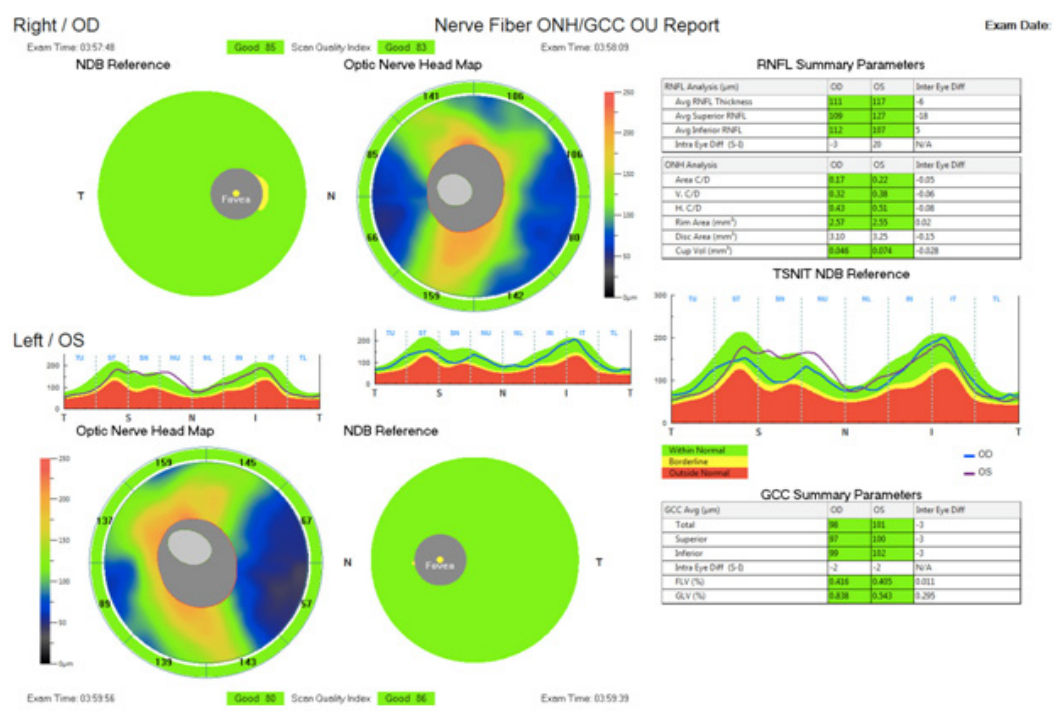

Figure 3 OCT images of the Macula (both eyes) and the Optic Nerve Head (Ganglion Cells Complex and Retinal Nerve Fiber Layer) (both eyes).

Skin biopsy was promptly performed from the cervical region. The histology report described elastic fibers that were irregular, thickened fragmented and haphazardly orientated in mid-dermis. Elastica VG and orcein stains verified their structure. The collagen fibers remained in a normal appearance. Calcium deposition was identified by von Kossa stain (Figure 4). Upon these findings, histological diagnosis of PXE was rendered.

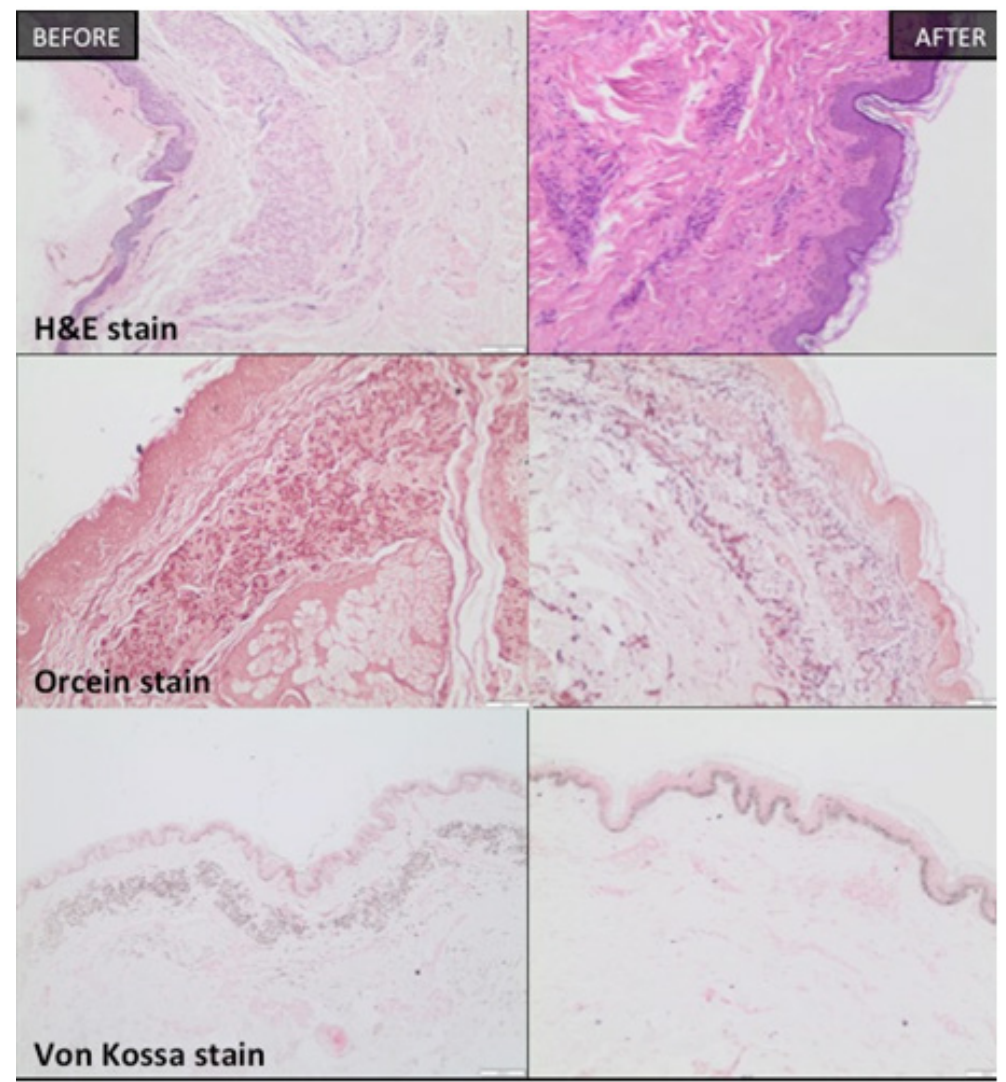

Figure 4 Neck Skin biopsies (H\&E, Elastica VG, orcein, Von Kossa stain).

The skin biopsy of the neck reveals the difference of the architecture of the elastin fibers with the parallel orientation and the high density (on the right) after the treatment protocol.Also, the calcium deposit on elastin fibers with von Kossa stain (on the left), has been almost disappeared after 3 sessions.

There was the possibility of performing a gene test of ABCC6 mutations. However, physician decided there was no need, due to highly characteristics of PXE of clinical and histologic features of lesions and ophthalmologic evidence as well. 


\section{Description of protocol - procedure}

Patient was fully informed about the description of neck rejuvenation protocol that was conducted in accordance with the Guidelines for Good Clinical Practice (GCP) and in compliance with the Declaration of Helsinki. Datas were anonymized prior to analysis and all clinical photographs were taken with respect to personal data protection regulations.

The Greek protocol for neck skin rejuvenation included 3 sessions of combined treatments, each comprising 3 different therapies:

\section{Fraxel Dual laser (1550// 927 nm)}

After a topical anesthetic (Xylocaine/Tetracaine), applied 1 hour priorly, the laser is operated in a scanning modefor skin resurfacing and mechanical trigger of ECM biosynthesis through creation of microscopic thermal zones of injury. Epidermal application of noncrosslinked hyaluronic acid is inserted through the created nanopores for better skin hydration. The first session was performed by1550$\mathrm{nm}$ erbium laser with pulse energy $45 \mathrm{~mJ}$ and level treatment 9 and then by $1927-\mathrm{nm}$ thulium laser with pulse energy $15 \mathrm{~mJ}$ and level treatment 3 . The second and third sessions were performed by $1550-$ $\mathrm{nm}$ erbium laser with pulse energy $55 \mathrm{~mJ}$ and level treatment 9 and then by $1927-\mathrm{nm}$ thulium laser with pulse energy $20 \mathrm{~mJ}$ and level treatment 4 . Each session included 8 overlapping passes, 4 per type of laser therapy. Forced cold air devices were used for surface cooling and improvement of patient tolerance. After each laser session, an erythema and edema were evident and cool hydration masks were placed on the neck region for extra cooling. A digital thermometer was used for skin temperature measurement. When room temperature was reached, the next step was followed in order to avoid protein coagulation.

\section{Type I horse collagen boosters}

After the addition of a small amount of $0,5 \mathrm{ml}$ xylocaine in the Nithya product, the latter was injected bi-laterally and symmetricallyby serial puncture technique with a 30 -gauge needle $(13 \mathrm{~mm})$. Type I horse collagen booster was intended to connect with fibroblasts' receptors for promoting collagen and elastin production.

\section{Platelet-rich plasma}

PRP, rich in various cytokines and growth factors, acts as molecular sign for fibroblasts' extracellular matrix synthesis as well as a promoter of cell proliferation and differentiation. Another mechanism of PRP for skin rejuvenation is acceleration of hyaluronic acid production. PRP is injected with a 30 -gauge needle (4 $\mathrm{mm}$ for superficial dermis and $6 \mathrm{~mm}$ for deep dermis), separately from collagen booster, in small injection points between the previous ones.

The above combination of therapies is based on the concept that skin rejuvenation needs the mechanical stimulus of laser-induced thermal injury of dermis as well as the scaffold of an appropriate microenvironment for fibroblasts. This microenvironment should include the precursors for fibroblasts (collagen boosters) as well as the necessary intercellular signaling (PRP) for cell proliferation and differentiation.

The protocol was repeated every 3 weeks for three times. During this period, the patient was encouraged for daily topical application of collagen mousse (3times/day) and vitamin C 35\% serum (every night) combined with a sunblock SPF50 (15-20 minutes before sunlight exposure). Due to COVID-19 quarantine time, her exposure to sunlight was limited.

\section{Results}

Clinical assessment supported by photographic documentation was performed each time, before every session. Impressive changes in hydration and skin tightening were obvious 3 weeks after the second session (Figure 5). Clinical enhancement was accompanied by histological improvement in a skin biopsy taken one month after the last session. The elastic fibers of the mid-dermis were normally oriented, less fragmented and much less calcified (Figure 4).

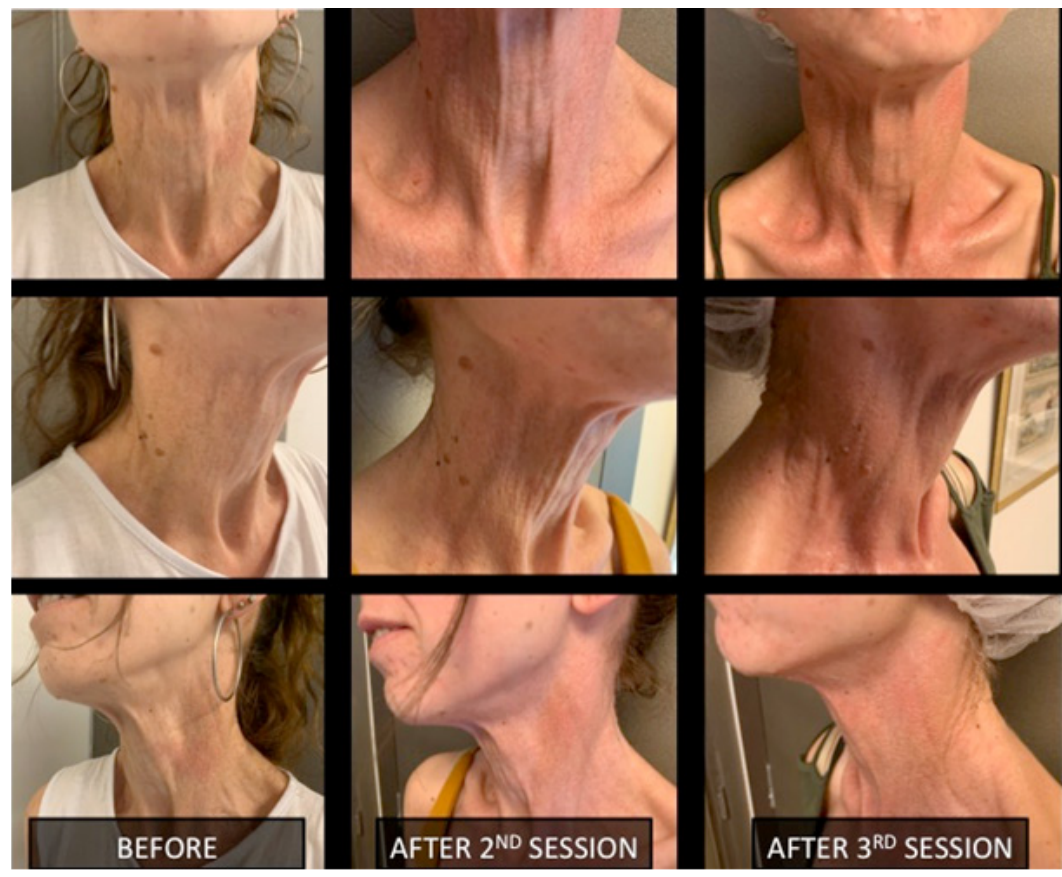

Figure 5 Neck Skin rejuvenation photos after $2^{\text {nd }}$ and $3^{\text {rd }}$ session.

The improvement of the texture of the neck skin with the tightness and the increased hydration of the area after 3 treatments. 
The aesthetic performance result persistent up to the final followup visit (12 months after), indicative of a long-lasting stimulatory effect on the cellular function (Figure 6).

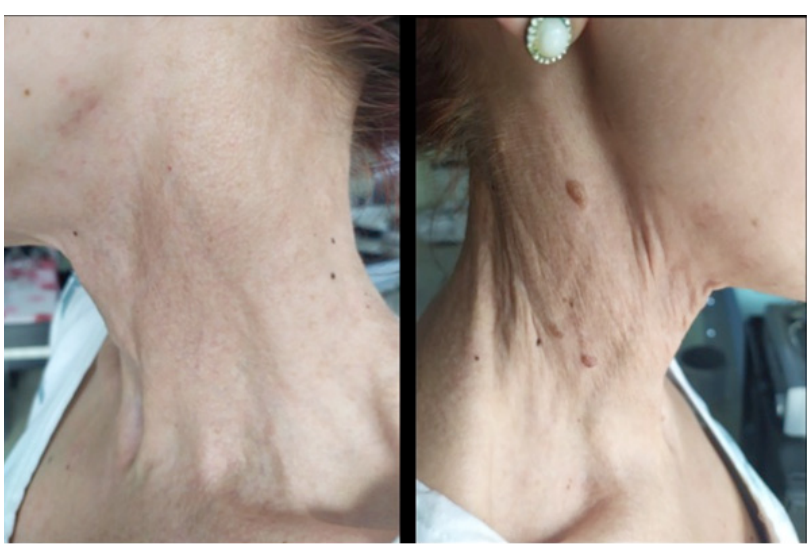

Figure 6 Postoperative clinical image of the neck 12 months after.

\section{Conclusions}

Regenerative medicine is an emerging field that focuses on cell- and tissue-based anti-aging skin therapies. The main goal of the up-to-date aesthetic medical research is oriented to the dermis bio-reconstruction. State-of-the-art treatments try to improve the dermis quality from inside-out in order to repair aging signs and imperfections. The key to success is attributed to the best possible treatment combination, capable of stimulating the fibroblasts. Fibroblasts need not only the mechanical trigger through skin resurfacing but also the appropriate microenvironment with the necessary precursor of collagen through biocompatible boosters and molecular signals by platelet-rich plasma. Inspired by this young woman with PXE with impressive, histologically and clinical verified results, this protocol is ready to be applicable as an anti-aging treatment for neck skin rejuvenation.

\section{Conflicts of interest}

The authors declare no conflict of interest related to this work.

\section{Funding}

None.

\section{Acknowledgments}

None.

\section{References}

1. Laube, S. Pseudoxanthoma elasticum. Arch Dis Child. 2005;90(7):754756.

2. Nitschke Y, Baujat G, Botschen U, et al. Generalized arterial calcification of infancy and pseudoxanthoma elasticum can be caused by mutations in either ENPP1 or ABCC6. Am J Hum Genet. 2012;90(1):25-39.

3. Marconi B, Bobyr I, Campanati A, et al. Pseudoxanthoma elasticum and skin: Clinical manifestations, histopathology, pathomechanism, perspectives of treatment. Intractable \& Rare Diseases Research. 2015;4(3);113-122.

4. Naouri, M, Boisseau C, Bonicel P, et al. Manifestations of pseudoxanthoma elasticum in childhood. British Journal of Dermatology. 2009;161(3):635639 .

5. Matsuo F, Berbert A, Mantese S, et al. Pseudoxanthoma Elasticum of the Skin with Involvement of the Oral Cavity. Case Reports in Dentistry. 2013:490785,1-6.
6. Issa P, Finger R, Götting C, et al. Centrifugal Fundus Abnormalities in Pseudoxanthoma Elasticum. Ophthalmology. 2010; 117(7):1406-1414.

7. Combrinck M, Gilbert J, Byard R. Pseudoxanthoma Elasticum and Sudden Death. J Forensic Sci. 2011;56(2):418-422.

8. Hyun J, Schwartz L, Walfish A. UGI Bleeding Secondary to Pseudoxanthoma Elasticum. American Journal of Gastroenterology. 2007;102, p.S318.

9. Hosen M, Lamoen A, De Paepe A, et al. Histopathology of Pseudoxanthoma Elasticum and Related Disorders: Histological Hallmarks and Diagnostic Clues. Scientifica. 2012;1-15.

10. Uitto J, Jiang Q, Váradi A, et al. Pseudoxanthoma Elasticum: Diagnostic Features, Classification, and treatment options. Expert Opin Orphan Drugs. 2014;2(6):567-577.

11. LaRusso J, Li Q, Jiang Q, et al. Elevated Dietary Magnesium Prevents Connective Tissue Mineralization in a Mouse Model of Pseudoxanthoma Elasticum (Abcc6---). Journal of Investigative Dermatology. 2009;129(6):1388-1394.

12. Bartstra J, de Jong P, Kranenburg G, et al. Etidronate halts systemic arterial calcification in pseudoxanthoma elasticum. Atherosclerosis. 2020;292:37-41.

13. Hendig D, Schulz V, Arndt M, et al. Role of Serum Fetuin-A, a Major Inhibitor of Systemic Calcification, in Pseudoxanthoma Elasticum. Clinical Chemistry. 2006;52(2):227-234.

14. Georgalas I, Tservakis I, Papaconstaninou D, et al. Pseudoxanthoma elasticum, ocular manifestations, complications and treatment. Clinical and Experimental Optometry. 2010;94(2):169-180.

15. Rongioletti F, Izakovic $\mathrm{J}$, Romanelli $\mathrm{P}$, et al. Pseudoxanthoma elasticum-like papillary dermal elastolysis: A large case series with clinicopathological correlation. J Am Acad Dermatol. 2012;67(1):128135.

16. Salles AG, Remigio AF, Moraes LB, et al. Pseudoxanthoma Elasticum Treatment with Fractional CO2 Laser. Plast Reconstr Surg Glob Open. 2014;2(9):e219.

17. Foering K, Torbeck R, Frank M. et al. Treatment of pseudoxanthoma elasticum-like papillary dermal elastolysis with nonablative fractional resurfacing laser resulting in clinical and histologic improvement in elastin and collagen. Journal of Cosmetic and Laser Therapy. 2018;20(78):382-384

18. Galadari H, Lebwohl M. Pseudoxanthoma elasticum: Temporary treatment of chin folds and lines with injectable collagen. Journal of the American Academy of Dermatology. 2003;49(5):265-266.

19. Ng A, O'Sullivan S, Sharpe D. Plastic surgery and pseudoxanthoma elasticum. British Journal of Plastic Surgery. 1999;52(7):594-596.

20. Quan T, Fisher G. Role of Age-Associated Alterations of the Dermal Extracellular Matrix Microenvironment in Human Skin Aging: A MiniReview. Gerontology. 2015;61(5):427-434.

21. Ricard-Blum S. The Collagen Family. Cold Spring Harbor Perspectives in Biology. 2010;3(1):a004978.

22. Weihermann A, Lorencini M, Brohem C, et al. Elastin structure and its involvement in skin photoageing. International Journal of Cosmetic Science. 2016;39(3):241-247.

23. Lee D, Oh J, Chung, J. Glycosaminoglycan and proteoglycan in skin aging. Journal of Dermatological Science. 2016;83(3):174-181.

24. Klein T, Bischoff R. Physiology and pathophysiology of matrix metalloproteases. Amino Acids. 2010;41(2):271-290.

25. Varga J, Rosenbloom J, Jimenez S. Transforming growth factor $\beta$ (TGF $\beta$ ) causes a persistent increase in steady-state amounts of type I and type III collagen and fibronectin mRNAs in normal human dermal fibroblasts. Biochemical Journal. 1987;247(3):597-604. 
26. Fisher G, Shao Y, He T, et al. Reduction of fibroblast size/mechanical force down-regulates TGF- $\beta$ type II receptor: implications for human skin aging. Aging Cell. 2015;15(1):67-76.

27. Shin J, Kwon S, Choi J, et al. Molecular Mechanisms of Dermal Aging and Antiaging Approaches. Int J of Moj Sci. 2019;20(9):2126.

28. Jih M, Kimyai-Asadi A. Fractional Photothermolysis: A Review and Update. Seminars in Cutaneous Medicine and Surgery. 2008;27(1):63-71.

29. Sparavigna A, Tateo A, Inselvini E, et al. Anti-Age Activity and Tolerance Evaluation of Collagen Micro-Injection Treatment Associated to Topical Application of a Cosmetic Formulation (Investigator-Initiated Multicentre Trial). Journal of Clinical \& Experimental Dermatology Research. 2017;08(03).
30. Puri N. Platelet rich plasma in dermatology and aesthetic medicine. Our Dermatology Online. 2015;6(2):207-211.

31. Kawashima S, Funakoshi T, Sato Y, et al. Protective effect of pre- and post-vitamin C treatments on UVB-irradiation-induced skin damage. Scientific Reports. 2018;8(1): 16199 